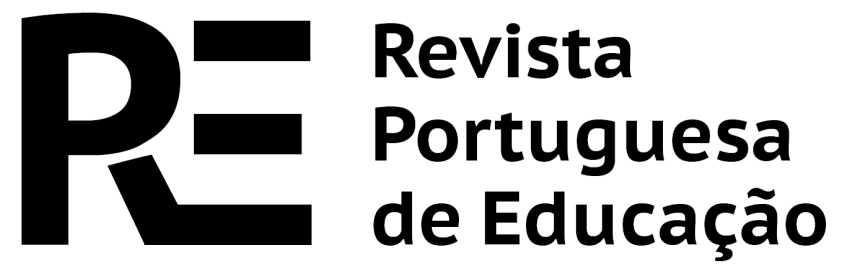

\title{
Desempenho escolar e diferentes papéis de participação no bullying: Um estudo transcultural
}

\begin{abstract}
RESUMO
O objetivo deste estudo é comparar o desempenho escolar entre os diferentes papéis de participação no bullying em dois países (Brasil e Portugal). Consiste num estudo transcultural de corte transversal de cunho exploratório e amostragem intencional. 376 crianças e adolescentes (do 5. ${ }^{\circ}$ e $6 .^{\circ}$ ano de escolas públicas e privadas) participaram deste estudo, sendo 197 de Portugal e 179 do Brasil. Os papéis de participação no bullying foram obtidos mediante a aplicação de uma escala sociométrica e o desempenho escolar foi avaliado pelas médias finais obtidas ao final do ano letivo de quatro disciplinas (ciências, inglês, matemática e português). Os dados foram analisados através da estatística descritiva e inferencial (teste Kruskal-Wallis e U de Mann-Whitney). Destaca-se que em Portugal as vítimas apresentaram melhor desempenho escolar. Já no Brasil não foram encontradas diferenças significativas. Os dados apontam que o desempenho escolar parece ser uma variável mais importante no estabelecimento dos papéis de participação no bullying em Portugal do que no Brasil, indicando que a relação entre essas variáveis não pode ser considerada característica padrão entre os envolvidos neste fenómeno em diferentes países.
\end{abstract}

Palavras-chave: Violência Escolar; Bullying; Desempenho Escolar

\section{INTRODUÇÃO}

Sabe-se que o bullying é um problema no âmbito educacional. 0 envolvimento neste fenómeno pode ser influenciado por fatores a nível individual, familiar, comunitário, cultural e principalmente escolar (Barboza et al.., 2009; Espelage \& de la Rue, 2013). Assim, fatores de risco e de proteção determinam o impacto do bullying para aqueles que participam da sua ocorrência. Na pesquisa de Khamis (2015), na qual foram analisados fatores individuais e contextuais relacionados à variância da gravidade do bullying, foi verificado que um ambiente escolar positivo, que favoreça a aprendizagem e incentive interações entre os estudantes baseadas na não
Marcela A. Zequinão ${ }^{i}$ Universidade do Estado de Santa Catarina, Brasil

Pâmella de Medeiros ${ }^{i i}$ Universidade do Estado de Santa Catarina, Brasil

Jorge Luiz da Silva ${ }^{\text {iii }}$ Universidade de Franca, Brasil

Luis Oliveira Lopes ${ }^{\text {iv }}$ Universidade do Minho, Portugal; Universidade do Porto, Portugal

Beatriz Oliveira Pereirav Universidade do Minho, Portugal 
violência, pode ser um fator de proteção para o não envolvimento nesse fenómeno.

No entanto, em muitos casos, a escola vem mostrando-se um local de produção e reprodução de violências e indisciplinas (Pinheiro et al., 2010; Silva \& Pereira, 2008), em que muitas crianças e adolescentes não se sentem seguros ou pertencentes a ela (Glew et al., 2008). Numa pesquisa nacional realizada nos Estados Unidos, verificou-se que todos os dias, quase 160.000 crianças não frequentam a escola porque têm medo de serem intimidadas, de acordo com a Associação Nacional de Educação (Rettew \& Pawlowski, 2016). No estudo de Glew et al. (2008), também nos Estados Unidos, 7\% dos estudantes que cursavam o $8^{\circ}$ ano, ficaram pelo menos um dia por mês em casa, em função do medo de outras crianças na escola. 0 medo da escola pode ser associado a um baixo desempenho escolar (Barboza, 2015), enquanto um clima escolar positivo pode melhorar a conquista académica dos escolares (Wang et al., 2014).

Assim, estudos produzidos na área da violência escolar vêm analisando a relação entre baixo desempenho e participação no bullying (Bowes et al., 2013; Chang et al., 2013; Costa \& Pereira, 2010; Krukowski et al., 2009; Kubwalo et al., 2013; Puhl \& King, 2013; Yang et al., 2013), evidenciando que este fenómeno causa efeitos negativos no desempenho escolar de crianças e adolescentes (Albdour \& Krouse, 2014; Barboza, 2015; Bilić et al., 2014; Copeland et al., 2013; Shirley \& Cornell, 2012). Contudo, a literatura é controversa em relação ao desempenho académico dos participantes que assumem diferentes papéis de participação no bullying. Alguns autores acreditam, por exemplo, que a vitimização pode ocasionar desempenho escolar deficiente, maior evasão escolar e absentismo (Anthony, Wessler \& Sebian, 2010; Cardoos \& Hinshaw, 2011; Cornell \& Mehta, 2011). Por outro lado, Smokowski e Kopasz (2005) identificaram os agressores com maiores problemas escolares, enquanto Gower et al. (2015) acreditam que, independentemente de ser agressor, vítima ou vítima-agressora, o envolvimento no bullying será prejudicial ao desempenho escolar.

A relação entre bullying e desempenho escolar é complexa, pois a intimidação representa apenas um dos fatores que pode contribuir para um baixo desempenho escolar. Desta forma, questiona-se: será que o desempenho escolar varia de acordo com os papéis de participação no bullying? Ou ainda, será que a relação entre desempenho escolar e bullying pode ser influenciada pelo contexto em que os participantes estão inseridos? Com o intuito de responder tais questionamentos o presente estudo teve como objetivo comparar o desempenho escolar entre os papéis de participação no bullying em dois países (Brasil e Portugal).

\section{MÉTODO}

\subsection{DESIGN}

Um estudo transcultural de corte transversal de cunho exploratório e amostragem intencional foi realizado na cidade metropolitana de Braga, na Região do Minho, ao Norte de Portugal, e também na cidade metropolitana de Florianópolis, estado de Santa Catarina, na Região Sul do Brasil, entre os meses de novembro de 2014 e maio de 2015. Esta pesquisa faz parte de um macroprojeto aprovada junto ao Comitê de Ética em Pesquisas com Seres Humanos da UDESC sob processo 5439/2011, $n^{\circ}$ expediente 75/2011 
(Brasil), e ao Comitê de Ética de Ciências Sociais e Humanas da Universidade do Minho, segundo o processo número 010/2014 (Portugal).

\subsection{PARTICIPANTES}

A seleção das escolas em ambos os países foi intencional, de acordo com aquelas que demonstraram interesse e disponibilidade em participar da pesquisa. Com isso, participaram do estudo quatro escolas públicas municipais e uma escola privada em Braga, e uma escola pública municipal e duas escolas privadas em Florianópolis. Após essa seleção, foram convidadas a participar todas as crianças e adolescentes do $5^{0}$ ao $6^{\circ}$ ano do Ensino Fundamental, de ambos os sexos, matriculadas no período vigente do estudo. Participaram deste estudo 376 participantes, 197 (156 de escola pública e 41 de escola privada) em Portugal e 179 (128 de escola pública e 51 de escola privada) no Brasil. Em termos de caracterização, apresentaram idade no intervalo compreendido entre 9 e 14 anos, média de 10,93 anos em Portugal e 10,81 anos no Brasil, distribuídos em $45,5 \%$ no $5^{\circ}$ ano e $54,5 \%$ no $6^{\circ}$ ano.

0 único critério de exclusão adotado no recrutamento dos participantes foi apresentar alguma deficiência intelectual que impedisse a compreensão do instrumento, com base na indicação do Atendimento Escolar Especial (AEE) das escolas.

\subsection{VARIÁVEIS}

O desempenho escolar foi avaliado pelas médias finais obtidas pelos alunos ao final do ano letivo. Foram selecionadas quatro disciplinas, as quais coincidiam em ambos os países nos respetivos anos escolares. As disciplinas foram: português, matemática, língua estrangeira (inglês) e ciências. Com as notas dessas quatro disciplinas também foi calculada uma média final para os participantes de ambos os países.

Já a participação no bullying, deu-se através de um exame sociométrico, denominado escala sociométrica (Levandoski \& Cardoso, 2013), que foi construído a partir de diversos outros estudos (Freire, Simão, \& Ferreira, 2006; Levandoski \& Cardoso, 2013; Olweus, 1994; Pereira et al., 2004; Smith, 2004). Este instrumento é constituído por perguntas do cotidiano da sala de aula relacionadas a comportamentos característicos de estudantes envolvidos em situações de bullying escolar, seja como vítima e/ou agressor. Cada participante indica o nome de três colegas de classe que mais se encontraram envolvidos nas situações descritas. Assim, todos os alunos tiveram dois escores, sendo um para agressão (somatório das questões 1, 3, $5,7,9$ e 11) e outro para vitimização (somatório das questões 2, 4, 6, 8, 10 e 12), gerados pelo número de vezes em que foram citados em cada bloco de perguntas. Como o número de participantes por sala variou uma em relação a outra, para estabelecer os escores utilizaram-se os valores padronizados pelo Escore Z. A partir desses escores, foi possível estabelecer uma classificação de participação no bullying, segundo o método estabelecido por Coie et al. (1982) para mensuração de medidas sociométricas. Neste método os participantes são qualificados como adequados para tipos extremos de status dentro do grupo de pares, sendo que esses tipos de status podem ser pensados em termos de setores dentro de uma grade bidimensional formado pela preferência social e impacto social. 0 método aponta as relações entre as dimensões de preferência social e impacto social e os cinco tipos de grupos de status que foram comparados no estudo de Coie et al. (1982), sendo que um grupo de status médio foi identificado para fornecer um grupo de 
referência com quem os grupos mais extremos poderiam ser comparados. A questão básica a ser abordada neste método diz respeito a diferenças potenciais (percebidas pelos pares) nos padrões de comportamento entre os cinco grupos de status.

Os nomes das categorias utilizadas por Coie et al. (1982) foram adaptadas nesse estudo, de acordo com os perfis de participação no bullying. Os participantes foram então classificados em cinco categorias: a) não participa - alunos que não foram citados pelos colegas ou aqueles citados de forma esporádica em situações de agressão ou vitimização (53,7\% dos participantes); b) grupo médio - alunos citados de maneira moderada em situações de agressão e vitimização, mas não de forma acentuada para serem classificados como um dos papéis de participação no bullying $(6,4 \%$ dos participantes); c) vítimas - alunos citados de forma acentuada para questões de vitimização 17,8\% dos participantes); d) agressores - alunos citados de forma acentuada para questões de agressão (11,2\% dos participantes); e) vítimas-agressoras - alunos que citados de forma acentuada para questões de vitimização e agressão (10,9\% dos participantes). Com o intuito de atender ao objetivo de identificar características dos papéis assumidos pelos envolvidos diretamente no bullying, os participantes do grupo médio foram excluídos das análises. A Escala Sociométrica apresentou um nível de consistência interna moderado, com um alfa de Cronbach igual a 0.744 .

\subsection{PROCEDIMENTOS}

Previamente à coleta dos dados, os pais e as crianças e adolescentes receberam informações detalhadas sobre a pesquisa. Os participantes foram devidamente esclarecidos sobre o estudo e explicitaram a sua anuência em participar da mesma através do Termo de Assentimento (TA), o qual foi elaborado em linguagem clara e acessível para os menores de idade. Da mesma forma foi enviado aos pais e/ou responsáveis o Termo de Consentimento Livre e Esclarecido (TCLE), documento no qual é explicitado o consentimento do responsável legal da criança. Após a entrega dos termos de assentimento e consentimento assinados, todos os participantes responderam individualmente à escala sociométrica (Levandoski, 2013) sobre a participação dos colegas no bullying escolar, na qual cada participante indicou quais eram os pares que vivenciavam mais situações como agressores e vítimas. Essa etapa foi aplicada e supervisionada por dois pesquisadores capacitados para esclarecer qualquer dúvida dos participantes. Ao final do ano letivo em cada país, foram recolhidas com a direção da escola as notas finais dos participantes naquele ano escolar, sendo que, no Brasil, as notas foram recolhidas em dezembro, enquanto em Portugal, as notas foram recolhidas no final de julho, em função dos calendários escolares serem diferentes em cada país.

\subsection{ANÁLISE ESTATÍSTICA}

Os dados foram analisados inicialmente pela estatística descritiva (média, desvio-padrão). A normalidade dos dados foi verificada através do teste Kolmogorov-Smirnov. A comparação das médias das variáveis foi realizada através do teste Kruskal-Wallis. Quando o teste foi significativo, comparações de dois em dois grupos foram realizadas para encontrar as diferenças, utilizando U de Mann-Whitney. Em todas as análises adotou-se o nível de significância de 5\%, utilizando o programa estatístico SPSS, versão 20.0. 


\section{RESULTADOS}

Quando analisados todos os participantes, verificaram-se diferenças significativas no desempenho escolar entre os papéis de participação no bullying em todas as disciplinas analisadas, bem como na média final. De acordo com os achados deste estudo, as vítimas apresentaram melhores resultados em todas as disciplinas quando comparadas aos outros papéis de participação no bullying ( $\mathrm{p}=0,001 ; \mathrm{p}=0,015 ; \mathrm{p}<0,001 ; \mathrm{p}=0,003 ; \mathrm{p}<0,001)$ (Tabela 1).

Tabela 1

Diferenças entre os papéis de participação no bullying em relação ao desempenho escolar, com todos os participantes

\begin{tabular}{|c|c|c|c|c|c|}
\hline \multirow[b]{2}{*}{ Variáveis } & \multicolumn{5}{|c|}{ TOTAL (N=376) } \\
\hline & $\begin{array}{c}\text { Não Participa } \\
\bar{x} \text { (DP) }\end{array}$ & $\begin{array}{l}\text { Vítima } \\
\bar{x} \text { (DP) }\end{array}$ & $\begin{array}{l}\text { Agressor } \\
\bar{x} \text { (DP) }\end{array}$ & $\begin{array}{c}\text { Vítima-Agressor } \\
\bar{x} \text { (DP) }\end{array}$ & p-valor* \\
\hline Matemática & $6,71(1,89)^{\mathrm{b}}$ & $7,52(1,69)^{\mathrm{a}}$ & $6,16(1,87)^{b}$ & $6,39(1,67)^{\mathrm{b}}$ & 0,001 \\
\hline Português & $6,75(1,65)^{\mathrm{b}}$ & $7,23(1,52)^{\mathrm{a}}$ & $6,49(1,45)^{\mathrm{b}}$ & $6,34(1,59)^{\mathrm{b}}$ & 0,015 \\
\hline $\begin{array}{l}\text { Língua Estrangeira } \\
\text { (Inglês) }\end{array}$ & $7,44(1,88)^{\mathrm{b}}$ & $8,31(1,56)^{\mathrm{a}}$ & $7,03(1,84)^{\mathrm{b}}$ & $7,03(1,94)^{\mathrm{b}}$ & $<0,001$ \\
\hline Ciências & $7,61(1,71)^{b}$ & $8,23(1,57)^{a}$ & $7,28(1,57)^{\mathrm{b}}$ & $7,21(1,53)^{b}$ & 0,003 \\
\hline Média Final & $7,13(1,61)^{b}$ & $7,82(1,35)^{a}$ & $6,74(1,47)^{\mathrm{b}}$ & $6,74(1,47)^{\mathrm{b}}$ & $<0,001$ \\
\hline
\end{tabular}

$\bar{X}$ (dp): média (desvio padrão); ${ }^{*}$ Kruskal-Wallis; ${ }^{\text {a-b }}$ Diferenças significativas identificadas pelo teste $U$ de Mann-Whitney

Ao analisar separadamente os participantes de Portugal, também foram encontradas diferenças significativas entre os papéis de participação no bullying em todas as médias analisadas. De acordo com os resultados, em português, as vítimas apresentaram melhor desempenho que os agressores e vítimas-agressoras $(\mathrm{p}=0,019)$. Já em matemática, língua estrangeira (inglês), ciências e média final, as vítimas apresentaram melhores resultados, tanto em relação aos agressores e vítimas-agressoras, quanto em relação aos que não participam do bullying $(\mathrm{p}=0,001 ; \mathrm{p}=0,010 ; \mathrm{p}=0,003 ; \mathrm{p}=0,001$, respetivamente) (Tabela 2 ).

Tabela 2

Diferenças entre os papéis de participação no bullying em relação ao desempenho escolar, com os participantes de Portugal

\begin{tabular}{lccccc}
\hline \multirow{2}{*}{ Variáveis } & \multicolumn{5}{c}{ PORTUGAL (N=197) } \\
\cline { 2 - 6 } & $\begin{array}{c}\text { Não Participa } \\
\bar{x}(\text { DP) }\end{array}$ & $\begin{array}{c}\text { Vítima } \\
\bar{x}(\text { DP) }\end{array}$ & $\begin{array}{c}\text { Agressor } \\
\bar{x}(\text { DP) }\end{array}$ & $\begin{array}{c}\text { Vítima- } \\
\text { Agressor } \\
\bar{x} \text { (DP) }\end{array}$ & $\begin{array}{c}\text { p- } \\
\text { valor }\end{array}$ \\
\hline Matemática & $6,52(1,83)^{\mathrm{b}}$ & $7,70(1,85)^{\mathrm{a}}$ & $5,92(2,04)^{\mathrm{b}}$ & $6,19(1,78)^{\mathrm{b}}$ & 0,001 \\
Português & $6,78(1,26)$ & $7,29(1,47)^{\mathrm{a}}$ & $6,48(1,45)$ & $6,19(1,78)^{\mathrm{b}}$ & 0,019 \\
Língua Estrangeira (Inglês) & $7,20(1,78)^{\mathrm{b}}$ & $8,20(1,74)^{\mathrm{a}}$ & $6,72(1,99)^{\mathrm{b}}$ & $6,67(2,39)^{\mathrm{b}}$ & 0,010 \\
Ciências & $7,84(1,65)^{\mathrm{b}}$ & $8,76(1,48)^{\mathrm{a}}$ & $7,44(1,68)^{\mathrm{b}}$ & $7,33(1,59)^{\mathrm{b}}$ & 0,003 \\
Média Final & $7,09(1,43)^{\mathrm{b}}$ & $7,99(1,40)^{\mathrm{a}}$ & $6,64(1,60)^{\mathrm{b}}$ & $6,59(1,70)^{\mathrm{b}}$ & 0,001 \\
\hline
\end{tabular}

$\bar{x}$ (dp): média (desvio padrão); ${ }^{*}$ Kruskal-Wallis; ${ }^{\text {a-b }} \mathrm{U}$ de Mann-Whitney 
Por fim, quando analisados os participantes brasileiros, não foram encontradas diferenças significativas entre o desempenho escolar e os papéis de participação no bullying (Tabela 3).

Tabela 3

Diferenças entre os papéis de participação no bullying em relação ao desempenho escolar, com os participantes do Brasil

\begin{tabular}{|c|c|c|c|c|c|}
\hline \multirow[b]{2}{*}{ Variáveis } & \multicolumn{5}{|c|}{$\begin{array}{c}\text { BRASIL } \\
(\mathrm{N}=179)\end{array}$} \\
\hline & $\begin{array}{l}\text { Não } \\
\text { Participa } \\
\bar{x} \text { (DP) }\end{array}$ & $\begin{array}{l}\text { Vítima } \\
\bar{x} \text { (DP) }\end{array}$ & $\begin{array}{r}\text { Agressor } \\
\bar{x} \text { (DP) }\end{array}$ & $\begin{array}{l}\text { Vítima- } \\
\text { Agressor } \\
\bar{x} \text { (DP) }\end{array}$ & p-valor \\
\hline Matemática & $6,91(1,93)$ & $7,33(1,50)$ & $6,52(1,57)$ & $6,59(1,56)$ & 0,290 \\
\hline Português & $6,72(1,99)$ & $7,17(1,61)$ & $6,50(1,49)$ & $6,50(1,38)$ & 0,358 \\
\hline $\begin{array}{l}\text { Língua Estrangeira } \\
\text { (Inglês) }\end{array}$ & $7,69(1,95)$ & $8,42(1,37)$ & $7,48(1,53)$ & $7,41(1,27)$ & 0,068 \\
\hline Ciências & $7,36(1,74)$ & $7,69(1,48)$ & $7,04(1,40)$ & $7,08(1,48)$ & 0,304 \\
\hline Média Final & $7,17(1,79)$ & $7,65(1,29)$ & $6,89(1,31)$ & $6,90(1,22)$ & 0,173 \\
\hline
\end{tabular}

$\bar{X}$ (dp): média (desvio padrão); ${ }^{*}$ Kruskal-Wallis; ${ }^{\text {a-b }} \mathrm{U}$ de Mann-Whitney

\section{DISCUSSÃO}

Este estudo teve como objetivo comparar o desempenho escolar entre os papéis de participação no bullying em dois países (Portugal e Brasil). Dentre os principais achados, destaca-se que, ao analisar todos os participantes de modo geral, as vítimas tiveram melhor desempenho escolar em todas as disciplinas quando comparadas aos agressores e vítimas-agressoras. Ao separar os participantes pelo país de origem, verificou-se que em Portugal as vítimas continuaram apresentando melhor desempenho em relação aos agressores e vítimas-agressoras, também em todas as disciplinas. Já no Brasil não foram encontradas diferenças significativas no que tange ao desempenho escolar entre os papéis de participação no bullying.

Estes resultados trazem uma nova perspetiva para os pesquisadores da área. A maior parte dos estudos relacionados com as variáveis desempenho escolar e bullying, apontam que baixo desempenho encontra-se associado à vitimização, parecendo haver um consenso na literatura sobre tal associação (Barboza, 2015; Busch et al., 2015; Lam et al., 2015; Wang et al., 2014). Alguns poucos estudos indicaram que desempenho escolar deficiente estava associado ao papel de agressor (Nansel et al., 2001; Smokowski \& Kopasz, 2005), ou que não foram encontradas associações entre desempenho escolar e envolvimento no bullying (Khamis, 2015). Contudo, os estudos que verificaram associações entre baixo desempenho escolar e o perfil de vítimas no bullying, ainda não foram capazes de afirmar se um baixo rendimento torna as crianças e os adolescentes mais propensos a tornarem-se vítimas pelos agressores, ou se, em função da violência sofrida, as vítimas tendem a ter uma queda no desempenho escolar. Todavia, os dados do presente estudo não confirmam essa associação, questionando o consenso dessa relação e as suas possíveis explicações, tendo em vista que os resultados apresentados inclinam as hipóteses para outra direção, nas quais a relação está entre melhor desempenho académico e vitimização em determinados contextos. 
Alguns estudos trazem evidências do que poderia explicar essa situação nesta pesquisa. Numa meta-análise realizada por Nakamoto e Schwartz (2010), para verificar as associações entre vitimização pelos pares e desempenho académico, foi encontrada uma associação negativa fraca, sugerindo que nenhuma conclusão ainda pode ser tirada em relação ao desempenho escolar inferior por parte das vítimas. De acordo com os pesquisadores, mais estudos são necessários, tendo em vista a existência de outras variáveis nessa relação que podem atuar ora omitindo, ora exacerbando tais associações. Para Bilić et al. (2014), apenas vitimização física foi associada a menor desempenho académico, o que vai ao encontro dos achados de Wang et al. (2014), os quais sugerem que nem toda vitimização terá um impacto negativo sobre as notas escolares, pois as diferenças na frequência e nos tipos de vitimização podem ser fatores determinantes para prever características do desempenho escolar de crianças e adolescentes vitimizados.

Por outro lado, no estudo de Al-Bitar et al. (2013), os autores destacaram que $35 \%$ dos participantes relataram sofrer algum tipo de bullying por ter notas boas. Essa informação vai ao encontro dos resultados deste estudo, no qual constatou-se melhor desempenho das vítimas em Portugal. Tais informações não permitem inferir a origem dessas relações, mas levam a criação de novas hipóteses para explicá-las. Assim, sugerem-se duas vertentes, uma na qual as vítimas podem buscar no desempenho escolar um reforço para superarem ou enfrentarem a vitimização sofrida, e outra seria que o fato das crianças e adolescentes obterem melhores resultados na escola faz com que eles se tornem alvos dos agressores.

Entretanto, não se pode afirmar que essa seria uma característica universal das vítimas de bullying, por exemplo, essa relação não foi encontrada neste estudo. Uma possível explicação para essa diferenciação entre estudos realizados nos dois países (Brasil e Portugal) pode estar relacionada à qualidade de ensino em cada país. Em grande parte, as situações de bullying ocorrem quando há uma disparidade entre os pares em relação a alguma variável, por exemplo, o nível socioeconômico que constitui um fator para a vitimização quando há crianças e adolescentes com diferentes níveis sociais dentro do mesmo contexto escolar. Desta forma, se pensarmos que a educação brasileira possui, por exemplo, a progressão continuada, em que não há reprovação em alguns anos escolares, talvez a variável desempenho escolar não seja tão importante para identificar o bullying no Brasil quanto é em Portugal.

A segunda hipótese é controversa na literatura que estuda a respeito dos sujeitos com altas habilidades/sobredotação. Por exemplo, Galvão (2009) destaca que os estudantes que possuem inteligência maior que a média da população, geralmente possuem dificuldades de relacionamentos sociais, timidez e falta de confiança, o que potencialmente os tornam alvos mais fáceis de bullying. Entretanto, Dalosto e Alencar (2013) identificaram que os estudantes com altas habilidades/sobredotação se envolviam em situações de bullying não apenas como vítimas, mas também como agressores e testemunhas. Esse estudo indica que um alto desempenho escolar ou um desenvolvimento intelectual superior não representa uma condição exclusiva para se tornar uma vítima de bullying.

Por outro lado, Wang et al. (2014) sugerem que vítimas de bullying em escolas com um clima escolar menos favorável (maior insegurança, fraco relacionamento entre professores e alunos, entre outros), tendem a apresentar notas mais baixas do que os pares de escolas com um 
clima escolar mais positivo. Contudo, independente de afetar ou não o desempenho escolar, sabe-se que a vitimização pelo bullying pode causar prejuízos em diversas outras esferas da vida dessas crianças e adolescentes (Bilić et al., 2014).

Entretanto, os achados dessa pesquisa ressaltam a importância de maior atenção por parte dos pesquisadores também aos outros participantes do bullying. Os resultados indicaram que agressores e vítimas-agressoras tendem a apresentar menores médias no que tange ao desempenho escolar. Sabe-se que agressores e vítimas-agressoras externalizam mais comportamentos problemáticos, o que pode contribuir para baixa competência académica (Barboza, 2015; Lam et al., 2015). De modo similar ao que acontece em relação à literatura sobre a vitimização, os estudos produzidos sobre a associação entre baixo desempenho escolar e comportamentos agressivos, também não são capazes de determinar as relações de causalidade entre as variáveis, em função da sua metodologia transversal (Zequinão et al., 2017).

Contudo, apesar de ainda não se poder afirmar que o baixo desempenho vulnerabiliza os estudantes à prática de agressões contra colegas na escola, ou que o envolvimento nessas situações prejudica o desempenho, sabe-se que, em grande parte, dificuldades de aprendizagem se apresentam associadas a outros problemas, principalmente de ordem comportamental e emocional (Elias, 2003; Martini \& Boruchovitch, 1999; Stevanato et al., 2003). As crianças com dificuldades de aprendizagem e de comportamento, geralmente caracterizam-se como menos envolvidas com as tarefas escolares do que os seus colegas sem dificuldades, além de constituírem um grupo vulnerável, com desenvolvimento prejudicado na adolescência (Santos \& Marturano, 1999; Stevanato et al., 2003), podendo ser um fator para o envolvimento no bullying escolar (Huepe et al., 2011; Lopes Neto, 2005). Assim, mais estudos visando analisar e compreender as características do desempenho escolar de agressores e vítimas-agressoras, bem como as relações de causa e efeito, são necessários.

Outro aspeto relevante deste estudo foi que o desempenho escolar parece ser uma característica mais importante na diferenciação dos envolvidos no bullying em Portugal do que no Brasil. A falta de estudos transculturais que investiguem as diferenças entre os países no que tange à relação entre desempenho escolar e bullying, limitam a interpretação dos resultados. Entretanto, em alguns estudos transculturais desenvolvidos a respeito do bullying, verificou-se que, independentemente do tipo de vitimização experimentada, nem todas as vítimas foram igualmente afetadas emocionalmente em diferentes países da Europa (Ortega et al., 2012), e que as diferenças de vitimização em crianças alemãs e inglesas podem ser explicadas por diferentes sistemas escolares em ambos os países (Wolke et al., 2001).

Com isso, acredita-se que tais diferenças encontradas nestes estudos transculturais, podem ser decorrentes de diferenças nas características culturais, nas políticas educacionais, entre as próprias escolas investigadas (Harel-Fisch et al., 2011; Smith-Khuri et al., 2004; Wolke et al., 2001) e nas rendas desiguais entre os países (Harel-Fisch et al., 2011), fatores que podem ter influência sobre fenómenos como o bullying. Assim, todos os envolvidos no meio escolar precisam identificar os fatores de risco e proteção associados a este fenómeno, para que seja possível estabelecer modelos mais sensíveis culturalmente, que contribuam para a avaliação, prevenção e intervenção do bullying (Khamis, 2015). Isso ressalta a relevância dos resultados do presente 
estudo, os quais apontam indícios de que o desempenho escolar pode ser uma variável importante nesse processo, principalmente em Portugal.

Como limitações desse estudo destacam-se: 1) a realização de uma pesquisa não probabilística não permite que os resultados encontrados possam ser estendidos de maneira geral a toda a população de ambos os países; 2) embora tenham sido encontradas algumas associações entre as variáveis analisadas, o delineamento transversal do estudo impede estabelecimento de causalidades. Pesquisas futuras podem adotar delineamento longitudinal em estudos transculturais, possibilitando o acompanhamento do fenómeno ao longo do tempo, de modo a compreenderem a relação entre desempenho escolar e os perfis dos participantes de bullying, identificando assim, relações de causa e efeito nos diferentes países.

\section{CONSIDERAÇÕES FINAIS}

Os resultados apresentados reforçam a relevância do bullying no ambiente educacional, pois confirmam as indicações de que ele se encontra presente em praticamente todas as escolas do mundo, independentemente de diferenças regionais ou culturais (Craig et al., 2009). Assim, identificar o bullying escolar nas suas diferentes formas de manifestação e o modo como afetam a vida das crianças e adolescentes, é fundamental para oferecer o suporte necessário. A esse respeito, em relação ao desempenho escolar, especificamente, os resultados identificados nesta investigação foram divergentes àqueles apresentados pela maior parte da literatura, por identificarem os estudantes portugueses vítimas de bullying com as melhores notas, quando comparados aos agressores e às vítimas-agressoras. Assim, os dados deste estudo indicam que o desempenho escolar parece ser uma variável mais importante no estabelecimento dos papéis de participação no bullying em Portugal do que no Brasil, demonstrando que a relação entre essas variáveis não pode ser considerada uma característica padrão entre os envolvidos neste fenómeno em diferentes países.

Os resultados obtidos neste estudo podem colaborar na elaboração de programas de intervenção que tenham como objetivo a promoção de boa convivência e de uma cultura de paz nas escolas. Intervenções direcionadas à prevenção e/ou ao enfrentamento do bullying escolar são importantes para auxiliar a modificar trajetórias escolares e de desenvolvimento pessoal dos estudantes que envolvam a participação em comportamentos violentos. 0 ideal, inclusive, é que tais intervenções estabelecendo redes integrais e articuladas de apoio e proteção, de modo a envolverem a equipe escolar na sua totalidade, as famílias dos estudantes e outros setores sociais como a saúde e os sistemas de proteção à criança e ao adolescente. É importante também considerar-se ações interventivas que sejam sensíveis às particularidades das diferentes realidades socioculturais nas quais as escolas estejam realizadas, tal como demonstrado pelos resultados deste estudo, de modo a serem mais contextualizadas e, portanto, mais efetivas.

\section{REFERÊNCIAS}

Al-Bitar, Z. B., Al-Omari, I. K., Sonbol, H. N., Al-Ahmad, H. T., \& Cunningham, S. J. (2013). Bullying among Jordanian schoolchildren, its effects on school performance, and the contribution of general physical and dentofacial 
features. Am J Orthod Dentofacial Orthop, 144(6), 872-878.

http://doi.org/10.1016/j.ajodo.2013.08.016

Albdour, M., \& Krouse, H. J. (2014). Bullying and victimization among African American adolescents: A literature review. J Child Adolesc Psychiatr Nurs, 27(2), 68-82. http://doi.org/10.1111/jcap.12066

Anthony, B. J., Wessler, S. L., \& Sebian, J. K. (2010). Commentary: Guiding a public health approach to bullying. J Pediatr Psychol, 35(10), 1113-1115. http://doi.org/10.1093/jpepsy/jsq083

Barboza, G. E. (2015). The association between school exclusion, delinquency and subtypes of cyber- and F2F-victimizations: Identifying and predicting risk profiles and subtypes using latent class analysis. Child Abuse Negl, 39, 109-122. http://doi.org/10.1016/j.chiabu.2014.08.007

Barboza, G. E., Schiamberg, L. B., Oehmke, J., Korzeniewski, S. J., Post, L. A., \& Heraux, C. G. (2009). Individual characteristics and the multiple contexts of adolescent bullying: Aan ecological perspective. J Youth Adolesc, 38(1), 101-121. http://doi.org/10.1007/s10964-008-9271-1

Bilić, V., Flander, G. B., \& Rafajac, B. (2014). Life satisfaction and school performance of children exposed to classic and cyber peer bullying. Coll Antropol, 38(1), 21-29.

Bowes, L., Maughan, B., Ball, H., Shakoor, S., Ouellet-Morin, I., Caspi, A., . . . Arseneault, L. (2013). Chronic bullying victimization across school transitions: The role of genetic and environmental influences. Dev Psychopathol, 25(2), 333-346. http://doi.org/10.1017/S0954579412001095

Busch, V., Laninga-Wijnen, L., Schrijvers, A. J., \& De Leeuw, J. R. (2015). Associations of health behaviors, school performance and psychosocial problems in adolescents in The Netherlands. Health Promot Int., 32(2), 280-291.

http://doi.org/10.1093/heapro/dav058

Cardoos, S. L., \& Hinshaw, S. P. (2011). Friendship as protection from peer victimization for girls with and without ADHD. J Abnorm Child Psychol, 39(7), 1035-1045. http://doi.org/10.1007/s10802-011-9517-3

Chang, F. C., Lee, C. M., Chiu, C. H., Hsi, W. Y., Huang, T. F., \& Pan, Y. C. (2013). Relationships among cyberbullying, school bullying, and mental health in Taiwanese adolescents. J Sch Health, 83(6), 454-462.

http://doi.org/10.1111/josh.12050

Coie, J. D., Dodge, K. A., \& Coppotelli, H. (1982). Dimensions and types of social status: A cross-age perspective. Developmental Psychology, 18, 557-570.

Copeland, W. E., Wolke, D., Angold, A., \& Costello, E. J. (2013). Adult psychiatric outcomes of bullying and being bullied by peers in childhood and adolescence. JAMA Psychiatry, 70(4), 419-426.

http://doi.org/10.1001/jamapsychiatry.2013.504

Cornell, D., \& Mehta, S. B. (2011). Counselor confirmation of middle school student self-reports of bullying victimization. Professional School Counseling, 14(4), 261-270.

Costa, P., \& Pereira, B. (2010). O bullying na escola: A prevalência e $o$ sucesso escolar. Paper presented at the I Seminário Internacional "Contributos da Psicologia em Contextos Educativos", Braga.

Craig, W., Harel-Fisch, Y., Fogel-Grinvald, H., Dostaler, S., Hetland, J., Simons-Morton, B., Molcho, M., Mato, M. G., Overpeck, M., Due, P., \& Pickett, W. (2009). A cross-national profile of bullying and victimization among adolescents in 40 countries. International Journal of Public Health, 54(2), 216-224.

https://doi.org/10.1007/s00038-009-5413-9 
Dalosto, M. M., \& Alencar, E. M. L. S. (2013). Manifestações e prevalência de bullyng entre alunos com altas habilidades/superdotação. Revista Brasileira de Educação Especial, 19, 363-378.

Elias, L. (2003). Crianças que apresentam baixo rendimento escolar e problemas de comportamento associados: Caracterização e intervenção [Doctoral dissertation, Universidade de São Paulo, Brasil].

https://repositorio.usp.br/item/001334977

Espelage, D. L., \& de la Rue, L. (2013). School bullying: Its nature and ecology. In J. C. Srabstein \& J. Merrick (Eds.), Bullying: A Public Health Concern (pp. 23-38). Nova Science Publishers

Freire, I., Simão, A., \& Ferreira, A. (2006). O estudo da violência entre pares no $3^{\circ}$ ciclo do ensino básico: Um questionário aferido para a população escolar portuguesa. Revista Portuguesa de Educação, 19(2), 157-183.

Galvão, D. P. (2009). Os nerds ganham poder e invadem a TV. Revista Intraciência, 001, 34-41.

Glew, G. M., Fan, M. Y., Katon, W., \& Rivara, F. P. (2008). Bullying and school safety. J Pediatr, 152(1), 123-128, 128.e121.

http://doi.org/10.1016/j.jpeds.2007.05.045

Gower, A. L., McMorris, B. J., \& Eisenberg, M. E. (2015). School-level contextual predictors of bullying and harassment experiences among adolescents. Soc Sci Med, 147, 47-53. http://doi.org/10.1016/j.socscimed.2015.10.036

Harel-Fisch, Y., Walsh, S. D., Fogel-Grinvald, H., Amitai, G., Pickett, W., Molcho, M., .. Group, M. o. t. H. V. a. I. P. F. (2011). Negative school perceptions and involvement in school bullying: A universal relationship across 40 countries. J Adolesc, 34(4), 639-652. http://doi.org/10.1016/j.adolescence.2010.09.008

Huepe, D., Roca, M., Salas, N., Canales-Johnson, A., Rivera-Rei, Á., Zamorano, L., . . I Ibañez, A. (2011). Fluid intelligence and psychosocial outcome: From logical problem solving to social adaptation. PLoS One, 6(9), e24858. http://doi.org/10.1371/journal.pone.0024858

Khamis, V. (2015). Bullying among school-age children in the greater Beirut area: Risk and protective factors. Child Abuse Negl, 39, 137-146. http://doi.org/10.1016/j.chiabu.2014.08.005

Krukowski, R. A., West, D. S., Philyaw Perez, A., Bursac, Z., Phillips, M. M., \& Raczynski, J. M. (2009). Overweight children, weight-based teasing and academic performance. Int J Pediatr Obes, 4(4), 274-280.

http://doi.org/10.3109/17477160902846203

Kubwalo, H., Muula, A. S., Siziya, S., Pasupulati, S., \& Rudatsikira, E. (2013). Prevalence and correlates of being bullied among in-school adolescents in Malawi: Results from the 2009 Global School-Based Health Survey. Malawi Med J, 25(1), 12-14.

Lam, S. F., Law, W., Chan, C. K., Wong, B. P., \& Zhang, X. (2015). A latent class growth analysis of school bullying and its social context: The selfdetermination theory perspective. Sch Psychol Q, 30(1), 75-90. http://doi.org/10.1037/spq0000067

Levandoski, G., \& Cardoso, F. (2013). Imagem corporal e status social de estudantes brasileiros envolvidos em bullying. Revista Latinoamericana de Psicología, 45(1), 135-145.

Lopes Neto, A. (2005). Bullying: Comportamento agressivo entre estudantes. J Pediatr (Rio J), 81(5), 164- 172.

Martini, M., \& Boruchovitch, E. (1999). As atribuições de causalidade, o desenvolvimento infantil e o contexto escolar. PSICO-USF, 4(2), 23-36. 
Nakamoto, J. \& Schwartz, D. (2010). Is peer victimization associated with academic achievement? A meta-analytic review. Social Development, 19, $221-$ 242. http://doi.org/10.1111/j.1467-9507.2009.00539.x

Nansel, T. R., Overpeck, M., Pilla, R. S., Ruan, W. J., Simons-Morton, B., \& Scheidt, P. (2001). Bullying behaviors among US youth: Prevalence and association with psychosocial adjustment. JAMA, 285(16), 2094-2100. http://doi.org/joc01746 [pii]

Olweus, D. (1994). Bullying at school: basic facts and effects of a school based intervention program. J Child Psychol Psychiatry, 35(7), 1171-1190.

Ortega, R., Elipe, P., Mora-Merchán, J. A., Genta, M. L., Brighi, A., Guarini, A., ... Tippett, N. (2012). The emotional impact of bullying and cyberbullying on victims: A European cross-national study. Aggress Behav, 38(5), 342-356. http://doi.org/10.1002/ab.21440

Pereira, B., Mendonça, D., Neto, C., Velente, L., \& Smith, P. (2004). Bullying in portuguese schools. School Psychology International, 25(2), 207-222.

Pinheiro, F., Stelko-Pereira, A., \& Williams, L. d. A. (2010). Bullying escolar: Caracterização dos alunos envolvidos, responsabilidade dos educadores e possibilidades de redução do problema. In G. d. E. d. Paraná \& S. d. E. d. Educação (Eds.), Enfrentamento à violência na escola (pp. 77-88). SEED.

Puhl, R. M., \& King, K. M. (2013). Weight discrimination and bullying. Best Pract Res Clin Endocrinol Metab, 27(2), 117-127. http://doi.org/10.1016/j.beem.2012.12.002

Rettew, D. C., \& Pawlowski, S. (2016). Bullying. Child Adolesc Psychiatr Clin N Am, 25(2), 235-242. http://doi.org/10.1016/j.chc.2015.12.002

Santos, L., \& Marturano, E. (1999). Crianças com dificuldades de aprendizagem: Um estudo de seguimento. Psicologia: Reflexão e Crítica, 12, 377-393.

Shirley, E. L., \& Cornell, D. (2012). The contribution of student perceptions of school climate to understanding the disproportionate punishment of African American students in a middle school. School Psychology International, 33(2), 115-134. http://doi.org/10.1177/0143034311406815

Silva, M., \& Pereira, B. (2008). A violência como fator de vulnerabilidade na ótica de adolescentes escolares. In J. Bonito (Coord.), Educação para a Saúde no Século XXI: Teorias, Modelos e Práticas. Universidade de Évora

Smith, P. (2004). Bullying: Recent developments. Child and Adolescent Mental Health, 9(3), 98-103.

Smith-Khuri, E., lachan, R., Scheidt, P. C., Overpeck, M. D., Gabhainn, S. N., Pickett, W., \& Harel, Y. (2004). A cross-national study of violence-related behaviors in adolescents. Arch Pediatr Adolesc Med, 158(6), 539-544.

http://doi.org/10.1001/archpedi.158.6.539

Smokowski, P. R., \& Kopasz, K. H. (2005). Bullying in school: An overview of types, effects, family characteristics, and intervention strategies. Children \& Schools, 27(2), 101-110. http://doi.org/10.1093/cs/27.2.101

Stevanato, I., Loureiro, S., Linhares, M., \& Marturano, E. (2003). Autoconceito de crianças com dificuldades de aprendizagem e problemas de comportamento. Psicologia em Estudo, 8(1), 67-76.

Wang, W., Vaillancourt, T., Brittain, H. L., McDougall, P., Krygsman, A., Smith, D., . . Hymel, S. (2014). School climate, peer victimization, and academic achievement: Results from a multi-informant study. Sch Psychol Q, 29(3), 360-377. http://doi.org/10.1037/spq0000084

Wolke, D., Woods, S., Stanford, K., \& Schulz, H. (2001). Bullying and victimization of primary school children in England and Germany: Prevalence and school factors. Br J Psychol, 92(Pt 4), 673-696. 
Yang, S. J., Stewart, R., Kim, J. M., Kim, S. W., Shin, I. S., Dewey, M. E., . . Yoon, J. S. (2013). Differences in predictors of traditional and cyber-bullying: A 2year longitudinal study in Korean school children. Eur Child Adolesc Psychiatry, 22(5), 309-318. http://doi.org/10.1007/s00787-012-0374-6

Zequinão, M. A., Cardoso, A. A., da Silva, J. L., de Medeiros, P., Silva, M. A. L., Pereira, B., \& Cardoso, F. L. (2017). Academic performance and bullying in socially vulnerable students. Journal of Human Growth and Development, 27(1), 19-27.

https://doi.org/10.7322/jhgd.127645

Informação dos autores:

i Centro de Ciências da Saúde e do Esporte, Universidade do Estado de Santa Catarina, Brasil.

https://orcid.org/0000-0003-3570-5425

ii Centro de Ciências da Saúde e do Esporte, Universidade do Estado de Santa Catarina, Brasil.

https: //orcid.org/0000-0001-9509-940X

iii Programa de Pós-Graduação em Promoção de Saúde, Universidade de Franca, Brasil.

https: //orcid.org/0000-0002-3727-8490

iv Instituto de Educação, Universidade do Minho, Portugal; Faculdade de Desporto, Universidade do Porto, Portugal. https://orcid.org/0000-0001-6680-0893

v Centro de Investigação em Estudos da Criança, Instituto de Educação, Universidade do Minho, Portugal.

https://orcid.org/0000-0003-4771-9402

Toda a correspondência relativa a este artigo deve ser enviada para:

Manuel Meira

Avenida Alcaides Faria, Edifício Santo António, 402H, Fração AU - 4750-106 Barcelos.

acoliveiramagmail.com 
School performance and different roles of participation in bullying: A cross-cultural study

\begin{abstract}
The relationship between bullying and school performance is a complex phenomenon and intimidation can contribute to poor school performance. The aim of this study was to compare school performance between the roles of involvement in bullying, segregated by country Portugal and Brazil. They participated in 785 children and adolescents. The participation in bullying roles, there was the couple indicated by Partner Scale Metric. Already the school performance was evaluated by the final averages at the end of the school year four disciplines. It is noteworthy that in Portugal the victims showed better school performance. In Brazil no significant differences were found. The data show that school performance seems to be a more important variable in establishing the bullying participation roles in Portugal than in Brazil, indicating that the relationship between these variables cannot be considered a standard feature among those involved in this phenomenon in different countries.
\end{abstract}

Keywords: School Violence; Bullying;

School Performance 
Desempeño escolar y diferentes papeles de participación en el acoso escolar: Un estudio transcultural

\section{RESUMEN}

Sufrir, practicar o presenciar situaciones de acoso escolar puede contribuir al bajo desempeño de los estudiantes. El objetivo de este estudio es comparar el rendimiento escolar entre los diferentes roles de participación en la intimidación en dos países (Brasil y Portugal). Las dos muestras fueron compuestas por 785 niños y adolescentes. Los roles de participación en la intimidación se obtuvieron mediante la aplicación de una escala sociométrico y el rendimiento escolar se evaluó mediante los promedios finales obtenidos al final del año escolar cuatro disciplinas (ciencias, inglés, matemáticas y portugués). Es de destacar que en Portugal las víctimas mostraron un mejor rendimiento escolar. En Brasil no se encontraron diferencias significativas. Los datos muestran que el rendimiento escolar parece ser una variable más importante para establecer los roles de participación en acoso escolar en Portugal que en Brasil, lo que indica que la relación entre estas variables no puede ser considerado como una característica estándar entre los involucrados en este fenómeno en diferentes países.

Palabras-clave: Violencia Escolar; Acoso Escolar; Rendimiento Escolar 